\title{
REVIEW
}

\section{Williams syndrome is an epigenome-regulator disease}

\author{
Hirochika Kitagawa $^{1), 2)}$, Ryoji Fujiki ${ }^{1), 3)}$, Kimihiro Yoshimura ${ }^{1), 3)}$, Hiroyuki Oya ${ }^{1)}$ and Shigeaki Kato ${ }^{1), 3)}$ \\ 1) Institute of Molecular and Cellular Biosciences, University of Tokyo, Tokyo 113-0032, Japan \\ 2) Institute for Molecular and Cellular Regulation, Gunma University, Maebashi 371-8512, Japan \\ 3) ERATO, Japan Science and Technology, Kawaguchi 332-0012, Japan
}

\begin{abstract}
A human multi-protein complex (WINAC), composed of SWI/SNF components and DNA replication-related factors, that directly interacts with the vitamin D receptor (VDR) through the Williams syndrome transcription factor (WSTF), was identified with an ATP-dependent chromatin remodeling activity. This novel ATP-dependent chromatin remodeling complex facilitates VDR-mediated transrepression as well as transactivation with its ATP-dependent chromatin remodeling activity and promoter targeting property for the activator to access to the DNA. It also suggested that in this complex, WSTF serves as a signaling sensor to receive intra-cellular singalings to switch the activity of WINAC as well as $\mathrm{WICH}$, another ATP-dependent chromatin remodeling complex containing hSNF2h. By making WSTF-deficient mice, some of the heart defects as well as abnormal calcium metabolism observed in Williams syndrome are attributed to the abnormal chromatin remodeling activity caused by WSTF deficiency. Thus, we would propose to designate Williams syndrome as an epigenome-regulator disease.
\end{abstract}

Key words: WSTF, VDR, Williams syndrome, Chromatin remodeling, Epigenome-regulator

CHROMATIN structure is intimately involved in the regulation of gene expression. The dynamics of chromatin structure are tightly regulated through multiple mechanisms such as histone modification, chromatin remodeling, histone variant incorporation, and histone eviction. Chromatin reorganization is performed by several chromatin-modifying complexes to allow effector proteins (transcription factors) access to DNA $[1,2]$. Two major classes of chromatin-modifying complexes have been well characterized [3]. One class is a histone-modifying complex, and the other class is an ATP-dependent chromatin-remodeling complex. The latter complex uses ATP hydrolysis to rearrange nucleosomal arrays in a non-covalent manner, thereby facilitating or preventing access of nuclear factors to nucleosomal DNA [2, 4]. Precise selection of catalytic ATPase subunits in combination with other components determines the role of these complexes in a spaciotemporal manner $[3,5]$. Studies of genetically

Received Dec. 15, 2010; Accepted Dec. 15, 2010 as K10E-393

Released online in J-STAGE as advance publication Jan. 14, 2011

Correspondence to: Hirochika Kitagawa, Institute of Molecular and Cellular Regulation, Gunma University, 3-39-15 Showa-Machi, Maebashi, Gunma, 371-8512, Japan.

E-mail: hirokita-tky@umin.ac.jp

(C)The Japan Endocrine Society altered animals deficient in individual complex-components suggest that each chromatin remodeling complex contributes to a specific cellular process. However, little is known about the regulatory mechanisms accounting for a complex's specific physiological impact [3, $6,7]$.

Williams syndrome (WS) is a rare autosomal dominant hereditary disorder with multiple symptoms, including typically congenital vascular lesion, elfin face, mental retardation, and growth deficiency [8]. Transient appearance of infantile aberrant vitamin D metabolism and hypercalcemia in the WS patients was also documented $[9,10]$. This syndrome is associated with genetic deletions at chromosome $7 \mathrm{q} 11.23$, and several candidate genes in the deleted regions have been mapped from their mRNA expression levels [11]. One gene, the Williams syndrome transcription factor (WSTF), has been suspected to be a candidate responsible for the diverse WS disorders $[8,12]$ (Fig.

Abbreviations: WSTF, Williams syndrome transcription factor; WINAC, WSTF Including Nucleosome Assembly Complex; WICH, WSTF-ISWI chromatin remodeling complex; VDR, Vitamin D receptor; SWI/SNF, SWItch/Sucrose NonFermentable; ISWI, Imitation Switch; BAF, BRG1 associated factors; ChIP, Chromatin Immunoprecipitation; MAPK, MAP-kinase 

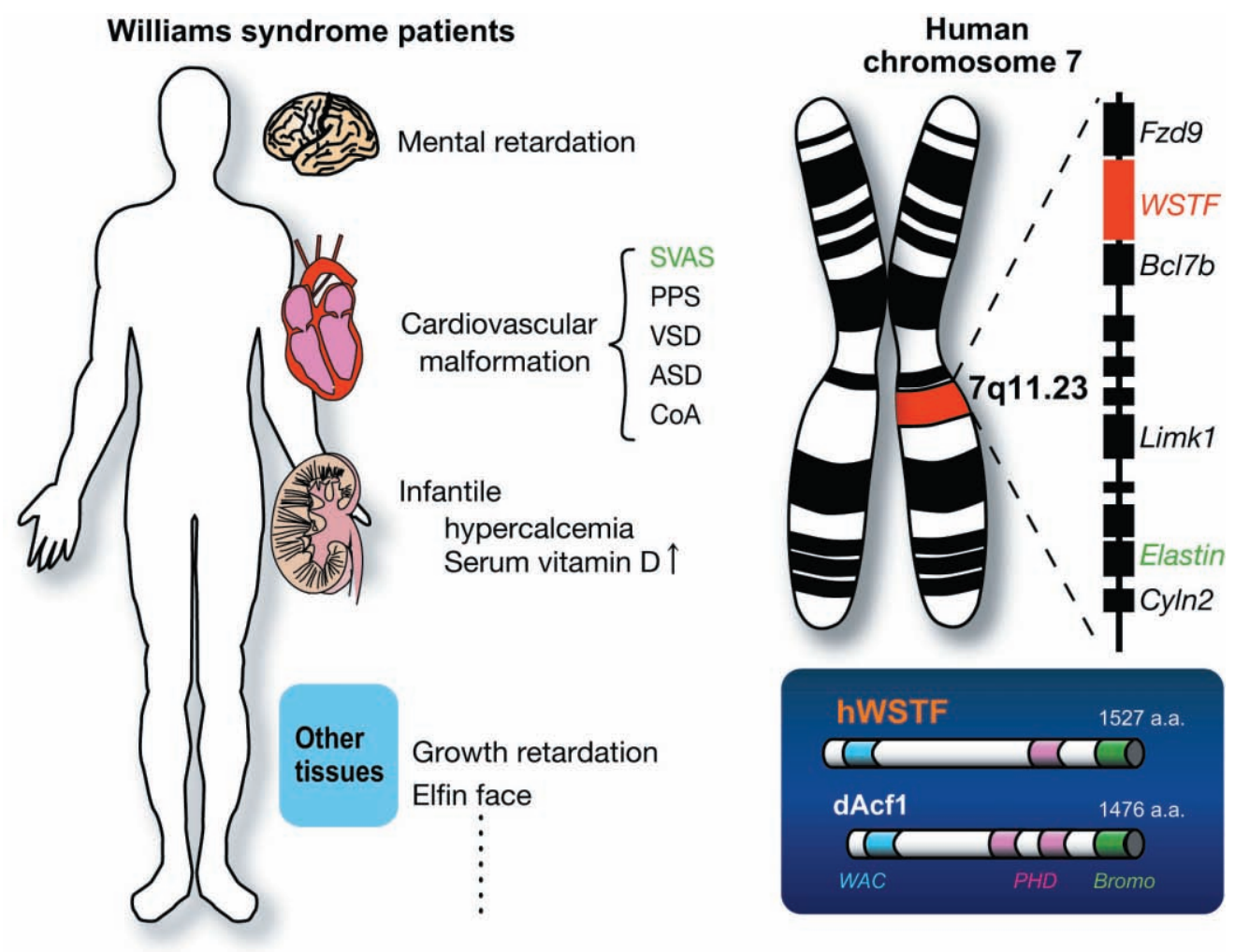

Fig. 1 Clinical manifestations of Williams syndrome and the structural characteristics of WSTF

(Left panel) Clinical manifestations of Williayms syndrome patients. (Right panel) WSTF is one of the candicate genes responsible for the etiology of Wiliams syndrome symptoms. (Right bottom panel) The characteristic domains contained in WSTF, such as Bromodomain, and PHD finger, can serve as a recognition motif for a certain chromatin structure

1). This possibility is raised by the fact that WSTF is highly homologous to hACF1 as one of the WAC family proteins [13]. Also, hACF1 is a partner of hSNF2h (a Drosophila ISWI homologue) to form well-characterized ISWI-based chromatin remodeling complexes [14] (Fig. 1: right bottom panel).

In the process of analyzing the transcriptional regulation mediated by Vitamin D receptor (VDR), we purified from MCF7 cells a novel human multiprotein complex named 'WINAC'. The analyses of WINAC represents not only a molecular mechanism how it regulates transcription mediated by some sequence-specific regulators with its chromatin remodeling activity, but also the relationship between the function of WINAC and Williams syndrome disorders $[15,16]$.

\section{WINAC is a Novel BAF-type ATP- Dependent Chromatin Remodeling Complex}

Chromatin structure is reorganized through chromatin remodeling and epigenetic modifications in the pro- cess of nuclear rearrangement. Two major classes of chromatin-modifying complexes that support nuclear events on chromosomes have been well characterized [17]. One class is a histone-modifying complex [18, 19], and the other class is an ATP-dependent chromatin-remodeling complex [1]. This complex uses ATP hydrolysis to rearrange nucleosomal arrays in a non-covalent manner to facilitate, or prevent, access of nuclear factors to nucleosomal DNA. These ATP-dependent chromatin-remodeling complexes have been classified into five subfamilies, the SWI/SNF-type complex, the ISWI-type complex, INO80 complex, SWR1 complex, and the NuRD-type complex. Each complex contains a major catalytic component that possesses DNAdependent ATPase activity, such as Brg-1/Brm (SWI/ SNF-type complex), or Snf2h (ISWI-type complex) $[20,21]$. Selection of catalytic ATPase subunits, combined with other complex components, defines the role of these complexes in various nuclear events including transcription, DNA replication, or DNA repair [7]. Previous genetical analyses have shown that the 


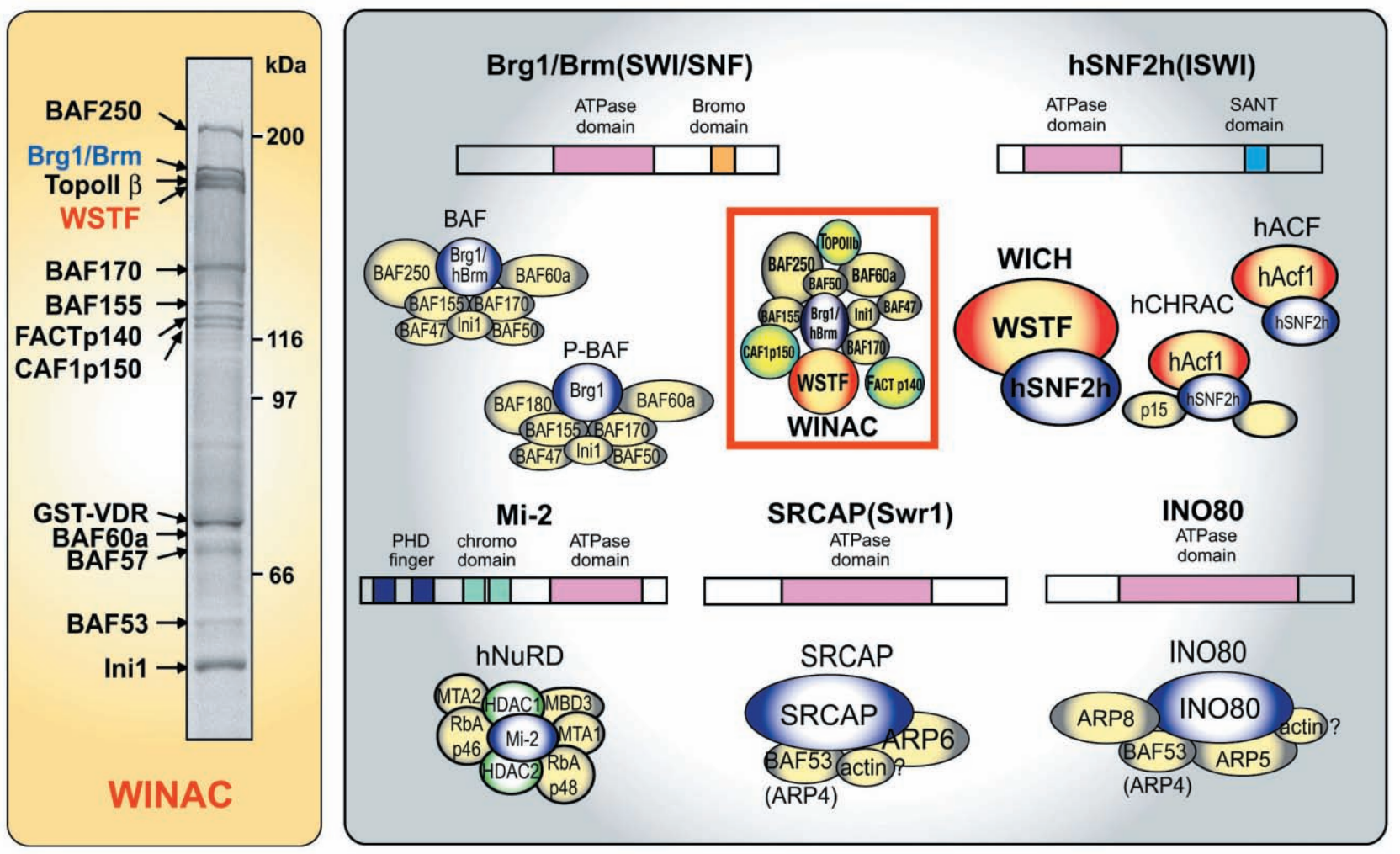

Fig. 2 The known ATP-dependent chromatin remodeling complexes

(Left panel) the identified WINAC components. (Right panel) WSTF is a component of SWI/SNF-type WINAC as well as ISWI-type WICH, which is composed of WSTF and hSNF2h (mammalian ISWI).

core-components of chromatin remodeling complexes are indispensable for embryonic development, while co-regulatory subunits appear to support the spatiotemporal function of the complexes [3, 22]. BAF60c was recently identified as a heart-specific subunit of the SWI/SNF-type complex [6] and the exchange of BAF45/53 subunits is essential for neuronal differentiation [23].

WSTF, one of the characteristic component of WINAC among SWI/SNF type complexes, is involved in BAZ family [13] (Fig. 2). Each member of this family is involved in distinct chromatin remodeling complexes and has distinct roles. WSTF contains a bromodomain and a PHD finger, which have been identified as a chromatin-recognition domain, but the function of this protein had been unknown. Besides, there is another WSTF-containing compelx designated as WICH, which is known to work as an ISWI-type chromatin remodeling complex especially works at DNA replication [14].

\section{WSTF is a Key Molecule for Transcriptional Repression as Well as Activation}

\section{Transcriptional Regulation mediated by Nuclear Receptor}

Lipophilic ligands, such as fat-soluble vitamins A and $\mathrm{D}$, as well as thyroid/steroid hormones, are thought to exert their physiological effects through transcriptional control of target genes via cognate nuclear receptors (NRs) [24]. NRs form a gene superfamily, and they act as ligand-inducible activators. A number of co-regulator complexes that support ligand-dependent transcription control have been identified, and these complexes can be classified into three categories according to function [25]. The first co-regulator complex class regulates transcriptional control directly, through a physical interaction with general transcription factors and RNA polymerase II [26]. Members of the second co-regulator complex class modify histone tails covalently, for example such as acetylation, and methylation in promoter nucleosomal arrays $[27,28]$. The 


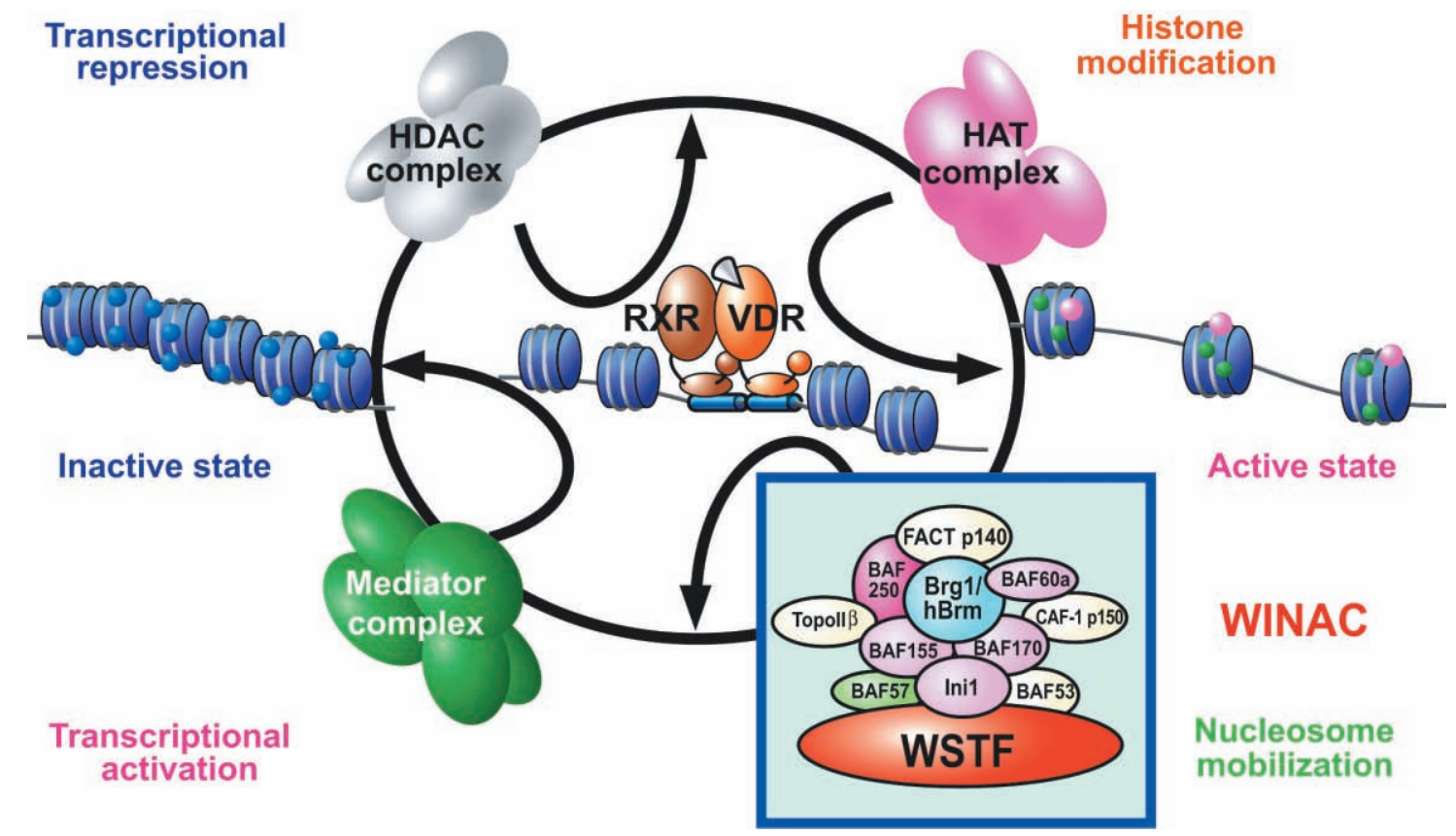

Fig. 3 Cyclic recruitment of the co-regulator complexes

Recently several types of chromatin remodeling complexes are known to be recruited in cyclically manner to the promoter regions in the process of ligand-dependent transcription mediated by nuclear receptors. WINAC takes part in this cyclic recruitment of co-regulator complexes in the VDR-mediated transcriptional process.

major function of the last class of complexes is chromatin remodeling, which involves the ATP-dependent dynamic remodeling of chromatin structure $[4,15]$.

The recent findings have deciphered that the each complex works in a 'hit and away' manner and the cyclic recruitment of each complex is generally observed when ChIP analysis is applied (Fig. 3).

\section{Transactivation and transrepression mediated by VDR}

Transcription control by NRs encompasses multiple steps with the help of a large number of co-regulator complexes $[25,29]$. ATP-dependent chromatin remodeling complexes are considered to support the promoterspecific recruitment of other co-regulator complexes [4].

Expression of the $25(\mathrm{OH}) \mathrm{D}_{3} \quad 1 \alpha$-hydroxylase $[1 \alpha(\mathrm{OH})$ ase $]$ gene, a key enzyme in vitamin $\mathrm{D}$ biosynthesis, is negatively regulated by vitamin $\mathrm{D}[30,31$, 32]. A bHLH-type activator, VDR-interacting repressor (VDIR), has been identified as a DNA-bound transcription factor the negative vitamin $\mathrm{D}$ response ele-

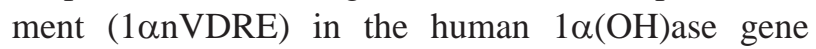
promoter, thus activating transcription [33]. However, ligand-induced association between VDR and VDIR results in ligand-induced transrepression of $1 \alpha(\mathrm{OH})$ ase gene expression. This occurs through the switching of co-regulator complexes from histone acetyltransferase (HAT) co-activator complexes to such co-repressors as histone deacetylase (HDAC), or DNA methylase, upon VDIR binding to $1 \alpha$ VDRE [33]. However, the molecular basis of VDR targeting to 1 $\alpha$ VDRE in the chromatinized $1 \alpha(\mathrm{OH})$ ase gene promoter remained unclear.

\section{Role of WINAC in the transrepression}

We investigated the function of WSTF in terms of the association between WINAC and chromatin for ligand-induced transrepression by VDR. Results of in vitro experiments using chromatin templates showed that the association of unliganded VDR with the promoter required physical interactions between WSTF and both VDR and acetylated histones prior to VDR association with chromatin. The acetylated histoneinteracting region of WSTF was mapped to the bromodomain, and a WSTF mutant lacking the bromodomain served as a dominant-negative mutant in terms of ligand-induced transrepression of the $1 \alpha(\mathrm{OH})$ ase gene. Thus, our findings indicate that WINAC associates with chromatin through a physical interaction between 


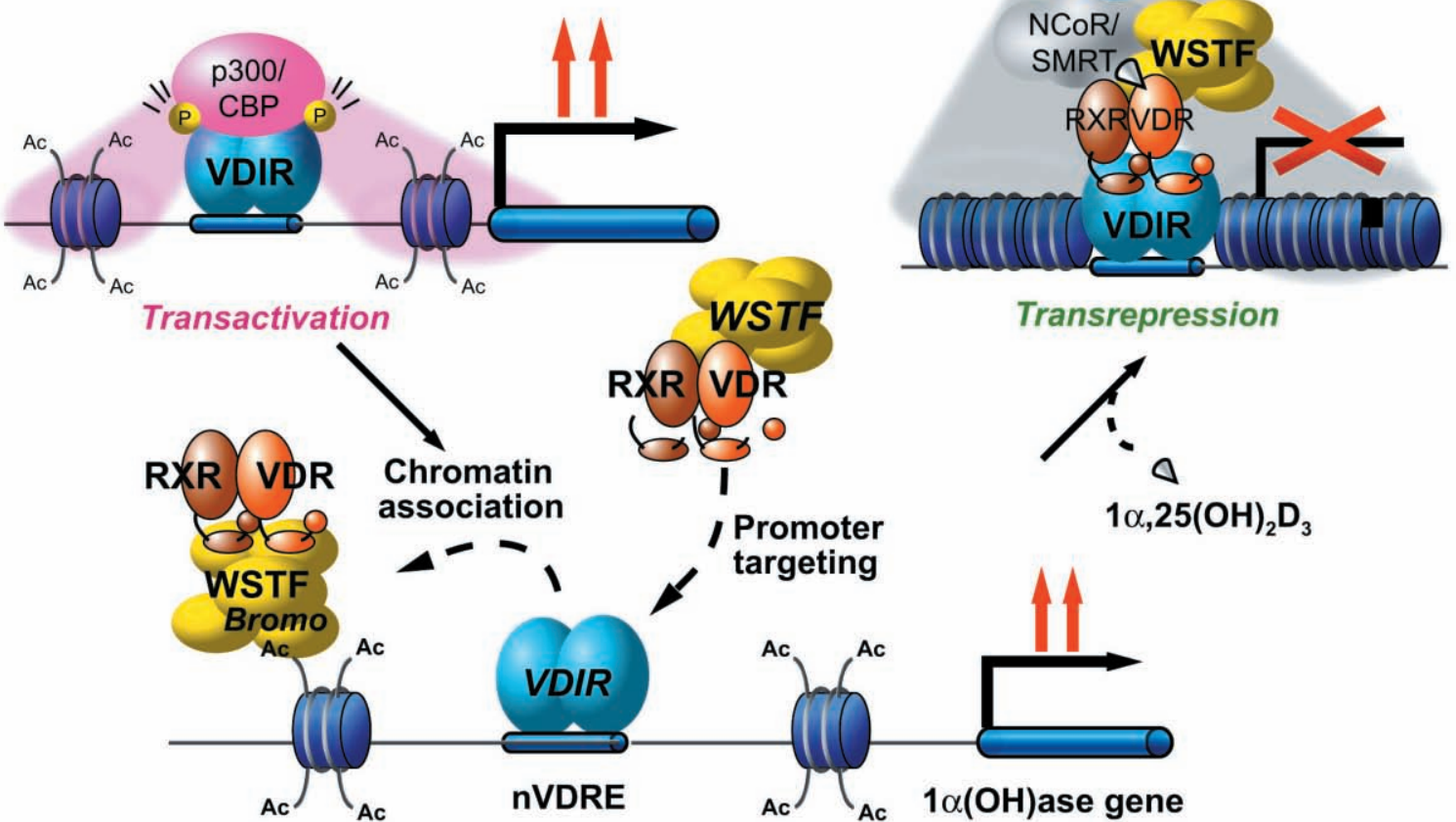

Fig. 4 The contribution of WINAC in the ligand-dependent transrepression

The role of WINAC in the lignad-dependent tranrepression is 1) Recognition of chromatin structure of the adjacent promoter regions and 2) promoter targeting of VDR to the negative VDRE.

the WSTF bromodomain and acetylated histones, that appears to be indispensable for VDR/promoter association for ligand-induced transrepression of $1 \alpha(\mathrm{OH})$ ase gene expression [34] (Fig. 4).

\section{Williams Syndrome as a Chromatin Remodeling Disease}

\section{Abnormal Calcium metabolism}

We found that the ligand-induced transactivation function of VDR is impaired in the skin fibroblast cells of the tested patients, in whom the regions covering the WSTF gene locus at the chromosome 7q 11.23 are heterozygously deleted. Such impaired VDR function should not lead to severe defects in vitamin $\mathrm{D}$ actions in adults, since the adult VDR heterozygote mice $\left(\mathrm{VDR}^{+/-}\right)$ and the heterozygous carrier patients of the hereditary vitamin D-dependent type II rickets caused by VDR inactivation exhibited no overt abnormality in calcium and vitamin D metabolism, though VDR is a major regulator in those metabolisms [30, 31]. However, during growth, the mineral intakes must be greater than their excretions through the actions of calciotropic hormones, including vitamin D. Thus it had been tempt- ing to speculate that the significantly reduced WINAC levels in WS patients transiently cause impaired function in VDR and other unidentified factors, leading to the transient appearance of infantile aberrant vitamin D metabolism and consequently, hypercalcemia $[9,10]$. These results were further supported by the observation in WSTF-deficient mice. Activated VDR by $1 \alpha, 25(\mathrm{OH})_{2} \mathrm{D}_{3}$ binding serves as a transcriptional repressor for $25(\mathrm{OH}) 1 \alpha$-hydroxylase gene $[1 \alpha(\mathrm{OH})$ ase] [31], while acts as an activator for 25(OH)24-hydroxylase [24(OH)ase] [15, 31, 34]. Although the upregulated expression of $1 \alpha(\mathrm{OH})$ ase, was observed, no clear induction of $24(\mathrm{OH})$ ase, was seen in spite of the excess $1 \alpha, 25(\mathrm{OH})_{2} \mathrm{D}_{3}$ observed in $\mathrm{WSTF}^{-/-}$embryos. Supporting these observations, serum calcium levels were elevated in the $\mathrm{WSTF}^{+/}$pups.

Taken together, Infantile hypercalcemia found in Williams syndrome patients thus appears attributed, at least in part, to malfunction of WSTF for a VDRmediated gene cascade $[9,15]$.

\section{Heart defect caused by the dysfunction of WINAC}

The physiological impacts of WSTF, including calcium metabolism were addressed by gene disruption 


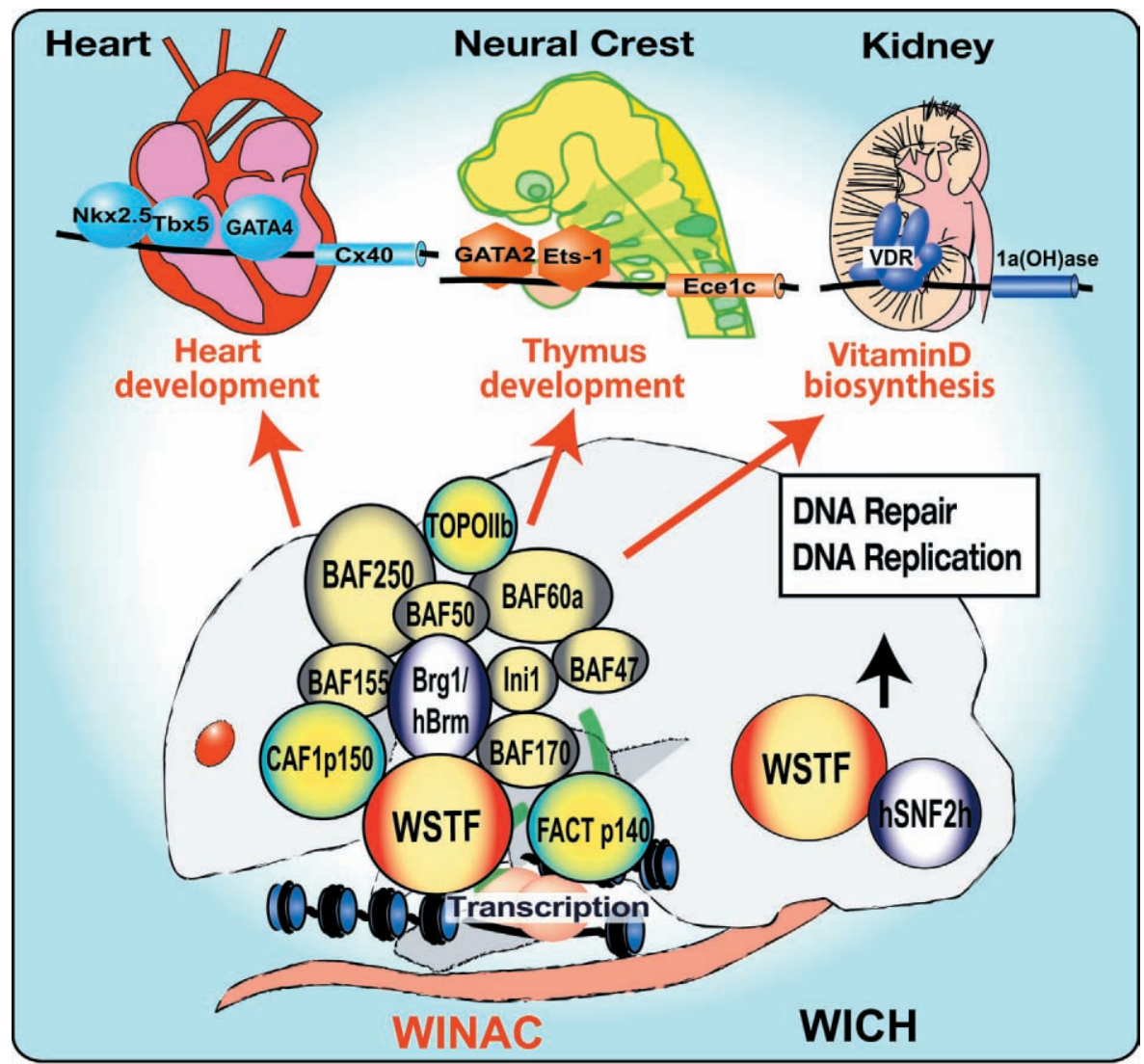

Fig. 5 Williams syndrome is a 'Chromatin remodeling disease'

Some symptoms observed in Williams syndrome patients are attributed to the dysfunction of WINAC or WICH. Thus Williams syndrome is caused by the aberrant chromatin remodeling activity, at least in part.

in mice [30]. WSTF-deficient mice were born with Mendelian frequency but died within a few days. They had smaller body sizes but normal appearance. Histological analysis of embryos revealed severe heart defects in all WSTF $^{-/-}$and $~ 10 \%$ of WSTF $^{+/-}$E9.5 embryos and neonates $(\mathrm{P} 0)$, which are reminiscent of the cardiac defects observed in Williams syndrome patients. Further analysis revealed that WSTF is a chromatin remodeler that is essential for physiological functions of certain sequence-specific transcriptional regulators which are essential for cardiac development. As we found that WSTF works as a component of WINAC in the transcription process, the abnormal genre expression observed in WSTF $^{-/-}$mice might presumably be caused by the dysfunction of WINAC (Fig. 5).

\section{Dysfunction of WICH}

Since WSTF is also a WICH component [14, 35], we suggest that certain phenotypes of $\mathrm{WSTF}^{-/-}$mice can be attributed to dysfunction of WICH. Although both WICH and WINAC were reported to affect DNA replication [14], no overt defects in the cell cycle or growth were found in WSTF $^{-/-}$MEF cells, as was seen in Xenopus egg [36]. Thus, WSTF appears dispensable for DNA replication in developing mice. Conversely, it is likely that WICH is indispensable for repair of DNA damage, since restored expression of WSTF, together with the WICH component Snf2h, ameliorated impaired survival after DNA damage in $\mathrm{WSTF}^{-/-}$ MEF cells.

In conclusion, the WSTF subunit appears to serve as a chromatin remodeler and is a component of two functionally-distinct complexes [16] (Fig. 5).

\section{WSTF Might Serve as a Signaling Sensor for Complex Switching}

Signal sensing of the chromatin remodeling complex

Intracellular signaling impacts gene regulation and thereby modulates cell proliferation, differentiation, 


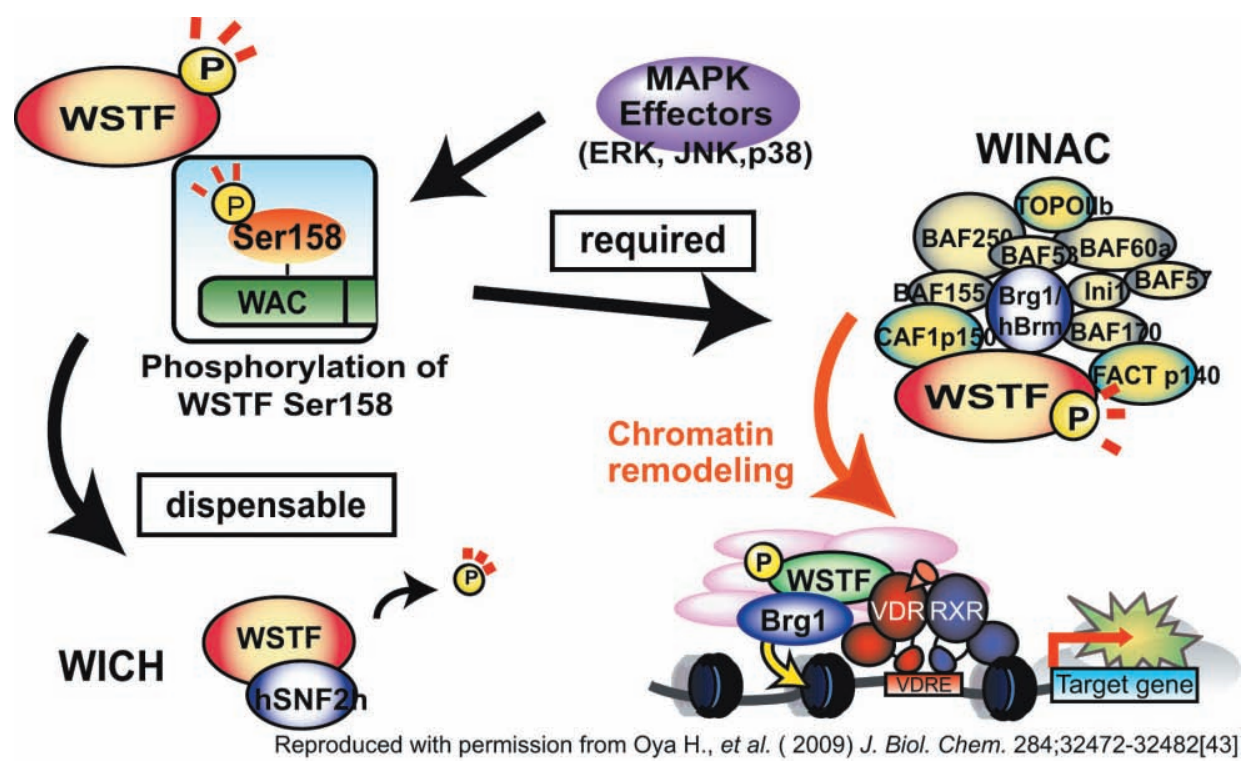

Fig. 6 WSTF possibly serves as a signaling sensor to switch two chromatin remodeling complexes, WINAC and WICH Phosphorylation of WSTF by MAP-kinases is necessary for the full activity of WINAC, but dispensable for WICH activity, suggesting a role of MAP-kinases to fine-tune the two chromatin remodeling complexes to work properly at specific conditions.

migration and apoptosis. Though several steps of the gene regulation can be modulated by various signaling molecules, the main regulatory mode is through modifications of transcription factors by their downstream effectors [37]. Several transcription factors including nuclear receptors have already been reported as modification targets $[38,39]$. But a detailed understanding of the regulatory mechanisms controlling transcription together with the reorganization of chromatin structure is lacking.

Chromatin remodeling complexes work at various situations to facilitate access of the biological effectors to the target regions of the genome through altering the adjacent chromatin status [40]. Considering the roles of the components specific for each chromatin remodeling complex [6, 23, 41, 42], we believe that WSTF, as a specific component of WINAC, works as a sensor of the various intracellular signalings for turning on its chromatin remodeling activity when required.

\section{MAPK-dependent regulation of WINAC function}

In our first screening, several inhibitors against various intracellular signaling cascades affected the co-activation function of WSTF. Further mechanical analysis seems essential to understand the biological impacts of WSTF as an epigenetic determinant under various extracellular stresses when distinct intracellular signaling cascades are activated.
Among them, we have described a novel epigenetic regulation by MAP-kinase (MAPK) cascades which modulate formation of an ATP-dependent chromatin remodeling complex, WINAC. WSTF was phosphorylated by the stimulation of MAPK cascades in vitro and in vivo. S158 residue in the WAC domain, located close to the N-terminus of WSTF, was identified as a major phosphorylation target. Using biochemical analysis of a WSTF mutant (S158A) stablyexpressing cell line, the phosphorylation of this residue (S158) was found to be essential for maintaining the association between WSTF and core BAF complexcomponents, thereby maintaining the ATPase activity of WINAC. WINAC-dependent transcriptional regulation of Vitamin D receptor (VDR) was consequently impaired by this WSTF mutation [43].

\section{Switching between WINAC and WICH}

But the recovery from DNA damage mediated by WICH was not impaired. Our results suggest that WSTF serves as a nuclear sensor of the extracellular signals to fine-tune the chromatin remodeling activity of WINAC, but not WICH. In other words, WINAC mediates a previously unknown MAPK-dependent step in epigenetic regulation and this MAPK-dependent switching mechanism between the two functionally distinct WSTFcontaining complexes might underlie the diverse functions of WSTF in various nuclear events [43] (Fig. 6). 


\section{Perspectives}

Recently, it is reported that WSTF itself works as a histone kinase during the repairing process after DNA damage when forming WICH complex [44]. Considering our data that WSTF is phosphorylated by MAP-kinases to switch the complex formation, it might be reasonable to speculate an unknown intra-cellular signalings can modulate the kinase activity of WSTF itself. In this sense, further analysis of the WSTF function might be interesting with consideration of the impact of WSTF in the physiological conditions.

As the WSTF-deficient mice die soon after birth, making spaciopemporally-specific WSTF-deficient mice should further contribute to the pathological analysis of Williams syndrome and this approach might lead to the development of a gene therapy to improve the lethal symptoms of the disease.

\section{Acknowledgement}

We would like to appreciate all the collaborators who helped finishing these sequential analysises. Also we would like to thank Ms. Chihiro Yajima for manuscript handling.

These works were partially supported by a grant from the Encouraging Development of Strategic Research Centers, Special Coordination Funds for Promoting Science and Technology, Ministry of Education, Culture, Sports, Science and Technology, Japan (to H.K. and S.K.).

\section{References}

1. Berger SL (2007) The complex language of chromatin regulation during transcription. Nature 447: 407-412.

2. Li B, Carey M, Workman JL (2007) The role of chromatin during transcription. Cell 128: 707-719.

3. de la Serna IL, Ohkawa Y, Imbalzano AN (2006) Chromatin remodelling in mammalian differentiation: lessons from ATP-dependent remodellers. Nat Rev Genet 7: 461-473.

4. Narlikar GJ, Fan HY, Kingston RE (2002) Cooperation between complexes that regulate chromatin structure and transcription. Cell 108: 475-487.

5. Emerson BM (2002) Specificity of gene regulation. Cell 109: 267-270.

6. Lickert H, Takeuchi JK, Von Both I, Walls JR, McAuliffe F, et al. (2004) Baf60c is essential for function of BAF chromatin remodelling complexes in heart development. Nature 432: 107-112.

7. Osley MA, Shen X (2006) Altering nucleosomes during DNA double-strand break repair in yeast. Trends Genet 22: 671-677.

8. Lu X, Meng X, Morris CA, Keating MT (1998) A novel human gene, WSTF, is deleted in Williams syndrome. Genomics 54: 241-249.

9. Garabedian M, Jacqz E, Guillozo H, Grimberg R, Guillot M, et al. (1985) Elevated plasma 1,25-dihydroxyvitamin D concentrations in infants with hypercalcemia and an elfin facies. $N$ Engl J Med 312: 948-952.

10. Taylor AB, Stern PH, Bell NH (1982) Abnormal regulation of circulating 25-hydroxyvitamin D in the Williams syndrome. $N$ Engl J Med 306: 972-975.

11. Hoogenraad CC, Koekkoek B, Akhmanova A, Krugers H, Dortland B, et al. (2002) Targeted mutation of Cyln2 in the Williams syndrome critical region links CLIP-115 haploinsufficiency to neurodevelopmental abnormalities in mice. Nat Genet 32: 116-127.

12. Peoples RJ, Cisco MJ, Kaplan P, Francke U (1998) Identification of the WBSCR9 gene, encoding a novel transcriptional regulator, in the Williams-Beuren syndrome deletion at 7q11.23. Cytogenet Cell Genet 82: 238-246.

13. Jones MH, Hamana N, Nezu J, Shimane M (2000) A novel family of bromodomain genes. Genomics 63: 40-45.

14. Poot RA, Bozhenok L, van den Berg DL, Steffensen S, Ferreira F, et al. (2004) The Williams syndrome transcription factor interacts with PCNA to target chromatin remodelling by ISWI to replication foci. Nat Cell Biol 6: 1236-1244.

15. Kitagawa H, Fujiki R, Yoshimura K, Mezaki Y, Uematsu Y, et al. (2003) The chromatin-remodeling complex WINAC targets a nuclear receptor to promoters and is impaired in Williams syndrome. Cell 113: 905-917.

16. Yoshimura K, Kitagawa H, Fujiki R, Tanabe M, Takezawa S, et al. (2009) Distinct function of 2 chromatin remodeling complexes that share a common subunit, Williams syndrome transcription factor (WSTF). Proc Natl Acad Sci U S A 106: 9280-9285.

17. Lee KK, Workman JL (2007) Histone acetyltransferase complexes: one size doesn't fit all. Nat Rev Mol Cell Biol 8: 284-295.

18. Klose RJ, Zhang Y (2007) Regulation of histone methylation by demethylimination and demethylation. Nat Rev Mol Cell Biol 8: 307-318.

19. Bannister AJ, Kouzarides T (2005) Reversing histone 
methylation. Nature 436: 1103-1106.

20. van Attikum H, Fritsch O, Hohn B, Gasser SM (2004) Recruitment of the INO80 complex by H2A phosphorylation links ATP-dependent chromatin remodeling with DNA double-strand break repair. Cell 119: 777-788.

21. Denslow SA, Wade PA (2007) The human Mi-2/NuRD complex and gene regulation. Oncogene 26: 54335438.

22. Bultman S, Gebuhr T, Yee D, La Mantia C, Nicholson J, et al. (2000) A Brg1 null mutation in the mouse reveals functional differences among mammalian SWI/SNF complexes. Mol Cell 6: 1287-1295.

23. Yoo AS, Staahl BT, Chen L, Crabtree GR (2009) MicroRNA-mediated switching of chromatin-remodelling complexes in neural development. Nature 460: 642-646.

24. Mangelsdorf DJ, Evans RM (1995) The RXR heterodimers and orphan receptors. Cell 83: 841-850.

25. Rosenfeld MG, Lunyak VV, Glass CK (2006) Sensors and signals: a coactivator/corepressor/epigenetic code for integrating signal-dependent programs of transcriptional response. Genes Dev 20: 1405-1428.

26. Freedman LP (1999) Increasing the complexity of coactivation in nuclear receptor signaling. Cell 97: 5-8.

27. Fujiki R, Chikanishi T, Hashiba W, Ito H, Takada I, et al. (2009) GlcNAcylation of a histone methyltransferase in retinoic-acid-induced granulopoiesis. Nature 459: 455459.

28. Yanagisawa J, Kitagawa H, Yanagida M, Wada O, Ogawa S, et al. (2002) Nuclear receptor function requires a TFTC-type histone acetyl transferase complex. Mol Cell 9: 553-562.

29. Glass CK, Ogawa S (2006) Combinatorial roles of nuclear receptors in inflammation and immunity. Nat Rev Immunol 6: 44-55.

30. Yoshizawa T, Handa Y, Uematsu Y, Takeda S, Sekine K, et al. (1997) Mice lacking the vitamin D receptor exhibit impaired bone formation, uterine hypoplasia and growth retardation after weaning. Nat Genet 16: 391-396.

31. Takeyama K, Kitanaka S, Sato T, Kobori M, Yanagisawa J, et al. (1997) 25-Hydroxyvitamin D3 1alpha-hydroxylase and vitamin D synthesis. Science 277: 1827-1830.

32. Murayama A, Takeyama K, Kitanaka S, Kodera Y, Hosoya T, et al. (1998) The promoter of the human 25-hydroxyvitamin D3 1 alpha-hydroxylase gene confers positive and negative responsiveness to PTH, calcitonin, and 1 alpha,25(OH)2D3. Biochem Biophys Res
Commun 249: 11-16.

33. Murayama A, Kim MS, Yanagisawa J, Takeyama K, Kato S (2004) Transrepression by a liganded nuclear receptor via a bHLH activator through co-regulator switching. EMBO J 23: 1598-1608.

34. Fujiki R, Kim MS, Sasaki Y, Yoshimura K, Kitagawa H, et al. (2005) Ligand-induced transrepression by VDR through association of WSTF with acetylated histones. EMBO J 24: 3881-3894.

35. Bozhenok L, Wade PA, Varga-Weisz P (2002) WSTFISWI chromatin remodeling complex targets heterochromatic replication foci. EMBO J 21: 2231-2241.

36. MacCallum DE, Losada A, Kobayashi R, Hirano T (2002) ISWI remodeling complexes in Xenopus egg extracts: identification as major chromosomal components that are regulated by INCENP-aurora B. Mol Biol Cell 13: 25-39.

37. Turjanski AG, Vaque JP, Gutkind JS (2007) MAP kinases and the control of nuclear events. Oncogene 26: 3240-3253.

38. Kato S, Endoh H, Masuhiro Y, Kitamoto T, Uchiyama S, et al. (1995) Activation of the estrogen receptor through phosphorylation by mitogen-activated protein kinase. Science 270: 1491-1494.

39. Pascual G, Fong AL, Ogawa S, Gamliel A, Li AC, et al. (2005) A SUMOylation-dependent pathway mediates transrepression of inflammatory response genes by PPAR-gamma. Nature 437: 759-763.

40. Sif S, Stukenberg PT, Kirschner MW, Kingston RE (1998) Mitotic inactivation of a human SWI/SNF chromatin remodeling complex. Genes Dev 12: 2842-2851.

41. Wang Z, Zhai W, Richardson JA, Olson EN, Meneses JJ, et al. (2004) Polybromo protein BAF180 functions in mammalian cardiac chamber maturation. Genes Dev 18: 3106-3116.

42. Takeuchi JK, Bruneau BG (2009) Directed transdifferentiation of mouse mesoderm to heart tissue by defined factors. Nature 459: 708-711.

43. Oya H, Yokoyama A, Yamaoka I, Fujiki R, Yonezawa M, et al. (2009) Phosphorylation of Williams syndrome transcription factor by MAPK induces a switching between two distinct chromatin remodeling complexes. J Biol Chem 284: 32472-32482.

44. Xiao A, Li H, Shechter D, Ahn SH, Fabrizio LA, et al. (2009) WSTF regulates the H2A.X DNA damage response via a novel tyrosine kinase activity. Nature 457: 57-62. 